\title{
Modeling of electromagnetic waves propagation in nonlinear optical media using HSCN-TLM method
}

\author{
Mohsine Khalladi $^{1 a)}$, M. I. Yaich ${ }^{1}$, N. Aknin ${ }^{1}$, and M. C. Carrión ${ }^{2}$ \\ ${ }^{1}$ Electronic \& Microwaves Group, \\ Faculty of Sciences, Abdelmalek Essaadi University, \\ P. O. Box: 2121, Tetuan 93000, Morocco \\ 2 Department of Applied Physics, \\ Faculty of Sciences, University of Granada, Spain \\ a)Khalladi@fst.ac.ma,M_khall@ieee.org
}

\begin{abstract}
In this paper, we propose a hybrid symmetrical condensed node TLM approach for the simulation of optical media under femtosecond regime. The formulation is based on the piecewise linear recursive convolution (PLRC) technique, voltage sources and the introduction of the variable admittance concept. Optical solitons with Kerr and Raman nonlinearities are simulated using this novel approach.
\end{abstract}

Keywords: nonlinear optical media, time-domain electromagnetics, TLM method

Classification: Electromagnetic theory

\section{References}

[1] M. I. Sobhy, M. W. R. Ng, R. J. Langley, and J. C. Batchelor, "TLM simulation of Ferrite media," Proc. 28th European Microwave Conf., Amesterdam, vol. 2, pp. 244-249, 1998.

[2] J. Paul, C. Christopoulos, and D. W. P. Thomas, "Generalized material models in TLM-Part 3: materials with nonlinear properties," IEEE Trans. Antennas Propagat., vol. 50, no. 7, pp. 997-1004, July 2002.

[3] D. M. Sullivan, "Non linear formulations using Z transforms," IEEE Trans. Microwave Theory Tech., vol. 43, pp. 676-682, March 1995.

[4] M. I. Yaich and Mohsine Khalladi, "The far-zone scattering calculation of frequency-dependent materials objects using the TLM method," IEEE Trans. Antennas Propagat., vol. 50, pp. 1605-1608, Nov. 2002.

[5] M. I. Yaich, M. Khalladi, I. Zekik, and J. A. Morente, "Modeling of frequency-dependent magnetized plasma in hybrid symmetrical condensed TLM method," IEEE Trans. Microwave Wireless Compon. Lett., vol. 12, pp. 293-295, Aug. 2002.

[6] M. I. Yaich and M. Khalladi, "A SCN-TLM Model for the Analysis of Ferrite Media," IEEE Trans. Microwave Wireless Compon. Lett., vol. 13, no. 6, pp. 217-219, June 2003.

[7] A. Taflove and S. C. Hagness, Computational Electrodynamics: The finite-difference time-domain method, 2nd ed., Artech House, 2000. 
[8] D. F. Kelley and R. Luebbers, "Piecewise linear recursive convolution for dispersive media using FDTD," IEEE Trans. Antennas propagat., vol. 44, pp. 792-797, 1996.

[9] P. M. Goorjian and A. Taflove, "Direct time integration of Maxwell's equations in nonlinear dispersive media for propagation and scattering of femtosecond electromagnetic solitons," Opt. Lett., vol. 17, pp. 180-182, Feb. 1992.

[10] C. Christopoulos, "The transmission-line modeling method TLM," IEEE Series on Electromagnetic Wave Theory, New York, 1995.

[11] H. Jin and R. Vahldieck, "Direct derivation of the TLM symmetrical condensed node and hybrid symmetrical condensed node from Maxwell's equations using centred differencing and averaging," Trans. Microwave Theory Tech., vol. 42, pp. 2554-2561, Dec. 1994.

[12] R. M. Joseph and A. Taflove, "FDTD Maxwell's equations models for nonlinear electrodynamics and optics," Trans. Antennas Propagat., vol. 45, no. 3, pp. 364-374, March 1997.

\section{Introduction}

Nonlinear optics field has experienced huge developments in recent years owing mainly to the great demand for faster and high performance communication systems. Along with this, there has been an increased need for accurate models allowing the electromagnetic (EM) field behavior in optical devices to be predicted for design purpose.

The recent developments of the transmission line matrix (TLM) method have shown its flexibility and efficiency for the simulation of EM waves propagation in complex media. This technique has been able to simulate EM wave propagation in dispersive anisotropic, dispersive bi-anisotropic and nonlinear media $[1,2,4,5,6]$. In this paper, a time domain approach for the simulation of nonlinear dispersive optical media using the TLM method is developed directly from Maxwell's equations and which does not make use of the Z-transform techniques [2, 3]. It consists in adding voltage sources modeling linear and nonlinear dispersive effects of the media in the hybrid symmetrical condensed node (HSCN), the use of the piecewise linear recursive convolution (PLRC) technique $[7,8]$ and the introduction of the variable admittance concept. The presented numerical results illustrate the propagation of EM waves in nonlinear Kerr and Raman media together with the linear Lorentzian dispersion.

\section{Formulation}

The PLRC-TLM formulation considering the hybrid symmetrical condensed node (HSCN) has been developed from the expression of the electric flux density $\mathrm{D}(\mathrm{t})$ given in [7]:

$$
\mathrm{D}(\mathrm{t})=\varepsilon_{0}\left[\varepsilon_{\infty} \mathrm{E}(\mathrm{t})+\mathrm{P}_{\mathrm{L}}(\mathrm{t})+\mathrm{E}(\mathrm{t})\left(\mathrm{P}_{\mathrm{NL}}(\mathrm{t})+\alpha_{0}^{(3)}(\mathrm{E}(\mathrm{t}))^{2}\right)\right]
$$

where $\varepsilon_{0}$ is the free space permittivity, $\varepsilon_{\infty}$ is the infinite frequency relative permittivity, $\alpha_{0}^{(3)}$ is a real constant defining Kerr's susceptibility, $\mathrm{E}(\mathrm{t})$ is the 
electric field such that the electric voltage $\mathrm{V}(\mathrm{t})=\mathrm{E}(\mathrm{t}) \Delta \mathrm{l}$ where $\Delta \mathrm{l}$ is the space step, $\mathrm{P}_{\mathrm{L}}$ and $\mathrm{P}_{\mathrm{NL}}$ are respectively the linear and the nonlinear polarisations. $\mathrm{P}_{\mathrm{L}}$ is expressed by the linear integral convolution of $\mathrm{E}(\mathrm{t})$ with the first order linear complex susceptibility $\hat{\chi}_{\mathrm{L}}^{(1)}(\mathrm{t})$ which contributes to the linear properties of the medium's frequency dependent permittivity, while $\mathrm{P}_{\mathrm{NL}}$ is given by nonlinear convolution integral of $\mathrm{E}(\mathrm{t})^{2}$ with the third order nonlinear complex susceptibility $\hat{\chi}_{\mathrm{NL}}^{(3)}(\mathrm{t})$ that models transient Raman scattering $[7$, 9], such that:

$$
\begin{aligned}
& \mathrm{P}_{\mathrm{L}}(\mathrm{t})=\frac{1}{\Delta \mathrm{l}} \int_{0}^{\mathrm{t}} \mathrm{V}(\tau) \chi_{\mathrm{L}}^{(1)}(\mathrm{t}-\tau) \mathrm{d} \tau \\
& \mathrm{P}_{\mathrm{NL}}(\mathrm{t})=\frac{1}{\Delta \mathrm{l}^{2}} \int_{0}^{\mathrm{t}}(\mathrm{V}(\tau)) \chi_{\mathrm{NL}}^{(3)}(\mathrm{t}-\tau) \mathrm{d} \tau
\end{aligned}
$$

where

$$
\begin{aligned}
& \chi_{\mathrm{L}}^{(1)}(\mathrm{t})=\operatorname{Re} \mathrm{al}\left(\hat{\chi}_{\mathrm{L}}^{(1)}(\mathrm{t})\right) \\
& \chi_{\mathrm{NL}}^{(3)}(\mathrm{t})=\operatorname{Re} \mathrm{al}\left(\hat{\chi}_{\mathrm{NL}}^{(3)}(\mathrm{t})\right)
\end{aligned}
$$

The complex susceptibilities $\hat{\chi}^{(1)}(\mathrm{t})$ and $\hat{\chi}^{(3)}(\mathrm{t})$ can be expressed in exponential form as follow:

$$
\begin{aligned}
& \hat{\chi}_{\mathrm{L}}^{(1)}(\mathrm{t})=\alpha_{\mathrm{L}} \mathrm{e}^{-\gamma_{\mathrm{L}} \mathrm{t}} \\
& \hat{\chi}_{\mathrm{L}}^{(3)}(\mathrm{t})=\alpha_{\mathrm{NL}} \mathrm{e}^{-\gamma_{\mathrm{NL}} \mathrm{t}}
\end{aligned}
$$

where $\alpha_{\mathrm{L}}, \alpha_{\mathrm{NL}}, \gamma_{\mathrm{L}}$ and $\gamma_{\mathrm{NL}}$ are complex magnitudes linked to the nonlinear properties of the medium. The use of the piecewise interpolation $[7,8]$ for $\mathrm{V}(\mathrm{t})$ and $(\mathrm{V}(\mathrm{t}))^{2}$ allows the time discretization of the convolutions appearing in Equations (2) and (3). We obtain at a time $\mathrm{t}=\mathrm{n} \Delta \mathrm{t}$ the following discrete expressions:

$$
\begin{aligned}
\mathrm{P}_{\mathrm{L}}= & \frac{1}{\Delta \mathrm{L}} \sum_{\mathrm{m}=0}^{\mathrm{n}-1}\left[\mathrm{~V}^{\mathrm{n}-\mathrm{m}} \chi_{\mathrm{L}}^{\mathrm{m}}-\left(\mathrm{V}^{\mathrm{n}-\mathrm{m}}-\mathrm{V}^{\mathrm{n}-\mathrm{m}-1}\right) \xi_{\mathrm{L}}^{\mathrm{m}}\right] \\
\mathrm{P}_{\mathrm{NL}}= & \frac{1}{\Delta \mathrm{L}^{2}} \sum_{\mathrm{m}=0}^{\mathrm{n}-1}\left[\mathrm{~V}^{\mathrm{n}-\mathrm{m}^{2}} \chi_{\mathrm{NL}}^{\mathrm{m}}-2 \mathrm{~V}^{\mathrm{n}-\mathrm{m}}\left(\mathrm{V}^{\mathrm{n}-\mathrm{m}}-\mathrm{V}^{\mathrm{n}-\mathrm{m}-1}\right) \xi_{\mathrm{NL}}^{\mathrm{m}}\right. \\
& \left.+\left(\mathrm{V}^{\mathrm{n}-\mathrm{m}-1}-\mathrm{V}^{\mathrm{n}-\mathrm{m}}\right)^{2} \zeta_{\mathrm{NL}}^{\mathrm{m}}\right]
\end{aligned}
$$

where $\mathrm{V}^{\mathrm{n}-\mathrm{m}}$ is the electric voltage at time $(\mathrm{n}-\mathrm{m}) \Delta \mathrm{t}$,

$$
\begin{aligned}
& \chi_{\mathrm{L}}^{\mathrm{m}}=\operatorname{Re} \mathrm{al}\left(\int_{\mathrm{m} \Delta \mathrm{t}}^{(\mathrm{m}+1) \Delta \mathrm{t}} \hat{\chi}_{\mathrm{L}}^{(1)}(\tau) \mathrm{d} \tau\right), \\
& \xi_{\mathrm{L}}^{\mathrm{m}}=\operatorname{Re} \mathrm{al}\left(\frac{1}{\Delta \mathrm{t}} \int_{\mathrm{m} \Delta \mathrm{t}}^{(\mathrm{m}+1) \Delta \mathrm{t}}(\tau-\mathrm{m} \Delta \mathrm{t}) \hat{\chi}_{\mathrm{L}}^{(1)}(\tau) \mathrm{d} \tau\right), \\
& \chi_{\mathrm{NL}}^{\mathrm{m}}=\operatorname{Re} \text { al }\left(\int_{\mathrm{m} \Delta \mathrm{t}}^{(\mathrm{m}+1) \Delta \mathrm{t}} \hat{\chi}_{\mathrm{NL}}^{(3)}(\tau) \mathrm{d} \tau\right),
\end{aligned}
$$




$$
\begin{aligned}
& \xi_{\mathrm{NL}}^{\mathrm{m}}=\operatorname{Re} \text { al }\left(\frac{1}{\Delta \mathrm{t}} \int_{\mathrm{m} \Delta \mathrm{t}}^{(\mathrm{m}+1) \Delta \mathrm{t}}(\tau-\mathrm{m} \Delta \mathrm{t}) \hat{\chi}_{\mathrm{NL}}^{(3)}(\tau) \mathrm{d} \tau\right), \\
& \zeta_{\mathrm{NL}}^{\mathrm{m}}=\operatorname{Re} \text { al }\left(\left(\frac{1}{\Delta \mathrm{t}}\right)^{2} \int_{\mathrm{m} \Delta \mathrm{t}}^{(\mathrm{m}+1) \Delta \mathrm{t}}(\tau-\mathrm{m} \Delta \mathrm{t})^{2} \hat{\chi}_{\mathrm{NL}}^{(3)}(\tau) \mathrm{d} \tau\right) .
\end{aligned}
$$

Using Equations (8) and (9) in Equation (1) gives the discrete time expression of the electric flux density at time $n \Delta t$ :

$$
\begin{aligned}
& \varepsilon_{0}^{\mathrm{n}}= \\
& {\left[\begin{array}{l}
\varepsilon_{\infty} \mathrm{V}^{\mathrm{n}}+\sum_{\mathrm{m}=0}^{\mathrm{n}-1}\left(\mathrm{~V}^{\mathrm{n}-\mathrm{m}} \chi_{\mathrm{L}}^{\mathrm{m}}-\left(\mathrm{V}^{\mathrm{n}-\mathrm{m}}-\mathrm{V}^{\mathrm{n}-\mathrm{m}-1}\right) \xi_{\mathrm{L}}^{\mathrm{m}}\right)+ \\
\frac{\mathrm{V}^{\mathrm{n}}}{\Delta \mathrm{L}^{2}} \sum_{\mathrm{m}=0}^{\mathrm{n}-1}\left[\begin{array}{l}
\mathrm{V}^{\mathrm{n}-\mathrm{m}^{2}} \chi_{\mathrm{NL}}^{\mathrm{m}}-2 \mathrm{~V}^{\mathrm{n}-\mathrm{m}}\left(\mathrm{V}^{\mathrm{n}-\mathrm{m}}-\mathrm{V}^{\mathrm{n}-\mathrm{m}-1}\right) \xi_{\mathrm{NL}}^{\mathrm{m}}+ \\
\left(\left(\mathrm{V}^{\mathrm{n}-\mathrm{m}-1}-\mathrm{V}^{\mathrm{n}-\mathrm{m}}\right)^{2} \zeta_{\mathrm{NL}}^{\mathrm{m}}\right)
\end{array}\right]+ \\
+\frac{\alpha_{0}^{(3)} \mathrm{V}^{\mathrm{n}^{3}}}{\Delta \mathrm{L}^{2}}
\end{array}\right]}
\end{aligned}
$$

The expression of the electric flux density $\mathrm{D}^{\mathrm{n}+1}$ at a time $(\mathrm{n}+1) \Delta \mathrm{t}$ is obtained following the same procedure. The knowledge of $\mathrm{D}^{\mathrm{n}}$ and $\mathrm{D}^{\mathrm{n}+1}$ represents a necessary and an indispensable step in the formulation and in the elaboration of the TLM algorithm allowing the simulation of the nonlinear optical media. The next step is based on Ampere's law in its discrete time form. Let's consider then an EM wave propagating in the z-direction, (i.e., $\mathrm{E}_{\mathrm{x}}$ and $\mathrm{H}_{\mathrm{y}}$ components) for which Ampere's law gives:

$$
(\nabla \times \mathrm{H})_{\mathrm{x}}^{\mathrm{n}+1 / 2}=\frac{\mathrm{D}_{\mathrm{x}}^{\mathrm{n}+1}-\mathrm{D}_{\mathrm{x}}^{\mathrm{n}}}{\Delta \mathrm{t}} .
$$

Developing Equation (16) by the use of the electric flux density expressions for $\mathrm{D}^{\mathrm{n}}$ and $\mathrm{D}^{\mathrm{n}+1}$, gives the following expressions for the electric voltage $\mathrm{V}_{\mathrm{x}}^{\mathrm{n}+1}$ at a time $(\mathrm{n}+1) \Delta \mathrm{t}$ :

$$
\mathrm{A}_{1} \mathrm{~V}_{\mathrm{x}}^{\mathrm{n}+1}+\mathrm{A}_{2} \mathrm{~V}_{\mathrm{x}}^{\mathrm{n}+1^{2}}+\mathrm{A}_{3} \mathrm{~V}_{\mathrm{x}}^{\mathrm{n}+1^{3}}=\mathrm{A}_{0} \mathrm{~V}_{\mathrm{x}}^{\mathrm{n}}+\left[\Delta \psi_{\mathrm{L}}^{\mathrm{n}}+\frac{\Delta \mathrm{t}}{\varepsilon_{0} \mathrm{Z}_{0}}(\nabla \times \mathrm{H})_{\mathrm{x}}^{\mathrm{n}+1 / 2}\right]
$$

where

$$
\begin{aligned}
& \mathrm{A}_{1}=\varepsilon_{\infty}+\chi_{\mathrm{L}}^{0}-\xi_{\mathrm{L}}^{0}+\mathrm{V}_{\mathrm{x}}^{\mathrm{n}^{2}} \zeta_{\mathrm{NL}}^{0} / \Delta \mathrm{L}^{2}+\psi_{\mathrm{NL}}^{\mathrm{n}+1} \\
& \mathrm{~A}_{2}=2 \mathrm{~V}_{\mathrm{x}}^{\mathrm{n}}\left(\xi_{\mathrm{NL}}^{0}-\zeta_{\mathrm{NL}}^{0}\right) / \Delta \mathrm{L}^{2} \\
& \mathrm{~A}_{3}=\left(\chi_{\mathrm{NL}}^{0}-2 \xi_{\mathrm{NL}}^{0}+\zeta_{\mathrm{NL}}^{0}+\alpha_{0}^{(3)}\right) / \Delta \mathrm{L}^{2} \\
& \mathrm{~A}_{0}=\varepsilon_{\infty}-\xi_{\mathrm{L}}^{0}+\psi_{\mathrm{NL}}^{\mathrm{n}}+\alpha_{0}^{(3)} \mathrm{V}_{\mathrm{x}}^{\mathrm{n}^{2}} / \Delta \mathrm{L}^{2} \\
& \Delta \psi_{\mathrm{L}}^{\mathrm{n}}=\sum_{\mathrm{m}=0}^{\mathrm{n}-1}\left[\mathrm{~V}_{\mathrm{x}}^{\mathrm{n}-\mathrm{m}}\left(\chi_{\mathrm{L}}^{\mathrm{m}}-\chi_{\mathrm{L}}^{\mathrm{m}+1}\right)-\left(\mathrm{V}_{\mathrm{x}}^{\mathrm{n}-\mathrm{m}}-\mathrm{V}_{\mathrm{x}}^{\mathrm{n}-\mathrm{m}-1}\right)\left(\xi_{\mathrm{L}}^{\mathrm{m}}-\xi_{\mathrm{L}}^{\mathrm{m}+1}\right)\right] \\
& \psi_{\mathrm{NL}}^{\mathrm{n}}=\frac{1}{\Delta \mathrm{L}^{2}} \sum_{\mathrm{m}=0}^{\mathrm{n}-1}\left[\left(\mathrm{~V}_{\mathrm{x}}^{\mathrm{n}-\mathrm{m}^{2}} \chi_{\mathrm{NL}}^{\mathrm{m}}-2 \mathrm{~V}_{\mathrm{x}}^{\mathrm{n}-\mathrm{m}}\left(\mathrm{V}_{\mathrm{x}}^{\mathrm{n}-\mathrm{m}}-\mathrm{V}_{\mathrm{x}}^{\mathrm{n}-\mathrm{m}-1}\right) \xi_{\mathrm{NL}}^{\mathrm{m}}+\mathrm{V}_{\mathrm{x}}^{\mathrm{n}-\mathrm{m}}\right)^{2} \zeta_{\mathrm{NL}}^{\mathrm{m}}\right. \\
& \psi_{\mathrm{NL}}^{\mathrm{n}+1}=\psi_{\mathrm{NL}}^{\mathrm{n}} \mathrm{e}^{-\gamma_{\mathrm{NL}} \Delta \mathrm{t}}
\end{aligned}
$$


Since the linear and nonlinear complex susceptibilities can be written in exponential form [7], the sums appearing in Equations (22) and (23) are computed recursively:

$$
\begin{aligned}
\Delta \psi_{\mathrm{L}}^{\mathrm{n}}= & {\left[\mathrm{V}_{\mathrm{x}}^{\mathrm{n}}\left(\chi_{\mathrm{L}}^{0}-\xi_{\mathrm{L}}^{0}\right)+\mathrm{V}_{\mathrm{x}}^{\mathrm{n}-1} \xi_{\mathrm{L}}^{0}\right]\left(1-\mathrm{e}^{-\gamma_{\mathrm{L}} \Delta \mathrm{t}}\right)+\mathrm{e}^{-\gamma_{\mathrm{L}} \Delta \mathrm{t}} \Delta \psi_{\mathrm{L}}^{\mathrm{n}-1} } \\
\psi_{\mathrm{NL}}^{\mathrm{n}}= & \frac{1}{\Delta \mathrm{L}^{2}}\left[\mathrm{~V}_{\mathrm{x}}^{\mathrm{n}^{2}} \chi_{\mathrm{NL}}^{0}-2 \mathrm{~V}_{\mathrm{x}}^{\mathrm{n}}\left(\mathrm{V}_{\mathrm{x}}^{\mathrm{n}}-\mathrm{V}_{\mathrm{x}}^{\mathrm{n}-1}\right) \xi_{\mathrm{NL}}^{0}+\left(\mathrm{V}_{\mathrm{x}}^{\mathrm{n}-1}-\mathrm{V}_{\mathrm{x}}^{\mathrm{n}}\right)^{2} \zeta_{\mathrm{NL}}^{0}\right] \\
& +\mathrm{e}^{-\gamma_{\mathrm{NL}} \Delta \mathrm{t}} \psi_{\mathrm{NL}}^{\mathrm{n}-1}
\end{aligned}
$$

Writing the magnetic field components of Equation (17) in terms of incident and reflected pulses $[10,11]$, then applying the charge conservation principle for the HSCN with a capacitive stub of normalized admittance $\mathrm{Y}_{\text {ox }}(\mathrm{t})$ we obtain the following equation:

$$
\begin{aligned}
& \mathrm{V}_{\mathrm{x}}^{\mathrm{n}+1}+\frac{\mathrm{A}_{2}}{\mathrm{~A}_{1}}\left(\mathrm{~V}_{\mathrm{x}}^{\mathrm{n}+1}\right)^{2}+\frac{\mathrm{A}_{3}}{\mathrm{~A}_{1}}\left(\mathrm{~V}_{\mathrm{x}}^{\mathrm{n}+1}\right)^{3} \\
& \quad=\frac{1}{2 \mathrm{~A}_{1}} \mathrm{n}+1 \\
&
\end{aligned}
$$

${ }_{n+1} V_{s x}$ is the voltage source introduced in port 16 of HSCN [5] in order to take into account in the TLM mesh linear and nonlinear dispersive properties and also Kerr effects and Raman interactions taking place in the nonlinear medium:

$$
{ }_{\mathrm{n}+1} \mathrm{~V}_{\mathrm{sx}}=-{ }_{\mathrm{n}} \mathrm{V}_{\mathrm{sx}}+4\left[\Delta \psi_{\mathrm{L}}^{\mathrm{n}}+\left(\mathrm{A}_{0}-\mathrm{A}_{1}\right) \mathrm{V}_{\mathrm{x}}^{\mathrm{n}}+\mathrm{A}_{2} \mathrm{~V}_{\mathrm{x}}^{\mathrm{n}^{2}}+\mathrm{A}_{3} \mathrm{~V}_{\mathrm{x}}^{\mathrm{n}^{3}}\right]
$$

$\mathrm{n}+1 \mathrm{Y}_{\mathrm{ox}}$ is the normalized admittance at a time $(\mathrm{n}+1) \Delta \mathrm{t}$ which is a function of the linear properties and which depends on the nonlinear properties and on the nature of the EM wave propagating in the nonlinear medium:

$$
{ }_{\mathrm{n}+1} \mathrm{Y}_{\mathrm{ox}}=4\left(\varepsilon_{\infty}+\chi_{\mathrm{L}}^{0}-\xi_{\mathrm{L}}^{0}+\left(\mathrm{V}_{\mathrm{x}}^{\mathrm{n}^{2}} \zeta_{\mathrm{NL}}^{0}\right) / \Delta \mathrm{L}^{2}+\psi_{\mathrm{NL}}^{\mathrm{n}+1}-1\right)
$$

To implement the PLRC-TLM algorithm with the HSCN and voltage sources, we compute at each iteration time the normalized admittance $\mathrm{Y}_{\mathrm{ox}}(\mathrm{t})$ given by Equation (29) and the voltage sources expressed by Equation (28). Then, we put the obtained values in the main Equation (27). This nonlinear equation is finally resolved using an iterative method. The solution of this equation is then invested in the computation of reflected pulses and in the connection process among the different TLM mesh nodes. All these operations require to memorize the two previous values for $\mathrm{V}_{\mathrm{x}}$.

\section{Numerical results}

To show the validity of this novel formulation, we consider a nonlinear optical medium with the following properties:

$$
\begin{array}{ll}
\alpha_{\mathrm{L}}=\mathrm{j} \frac{\varepsilon_{\mathrm{s}}-\varepsilon_{\infty}}{\sqrt{\omega_{0}^{2}-\delta^{2}}} \omega_{0}^{2}, & \gamma_{\mathrm{L}}=\delta+\mathrm{j} \sqrt{\omega_{0}^{2}-\delta^{2}}, \\
\alpha_{\mathrm{NL}}=\mathrm{j} \frac{\tau_{1}^{2}+\tau_{2}^{2}}{\tau_{1} \tau_{2}^{2}} \chi_{0}^{(3)}, & \gamma_{\mathrm{NL}}=\frac{1}{\tau_{2}}+\mathrm{j} \frac{1}{\tau_{1}},
\end{array}
$$


where $\varepsilon_{\mathrm{S}}$ is the static permittivity, $\omega_{0}$ the resonant frequency and $\delta$ is the damping coefficient. $\left(1 / \tau_{1}\right)$ is the optical phonon frequency, $\left(1 / \tau_{2}\right)$ is the phonon bandwidth and $\chi_{0}^{(3)}$ is the nonlinear coefficient.

The values of the parameters of the analyzed medium are [7]: $\varepsilon_{\mathrm{S}}=$ $5.25 ; \varepsilon_{\infty}=2.25 ; \omega_{0}=4.0 \times 10^{14} \mathrm{rad} / \mathrm{s} ; \delta=2.0 \times 10^{9} \mathrm{~s}^{-1}, \tau_{1}=12.2 \mathrm{fs} ;$ $\tau_{2}=32.0 \mathrm{fs} ; \chi_{0}^{(3)}=21.0 \times 10^{-3} \mathrm{~V}^{-2}$ and $\alpha_{0}^{(3)}=49.0 \times 10^{-3} \mathrm{~V}^{-2}$. The considered TLM mesh dimensions are $(1,1,8000) \Delta \mathrm{l}$ with $\Delta \mathrm{l}=25 \cdot 010^{-9} \mathrm{~m}$. The air-nonlinear dispersive medium interface is located at $\mathrm{z}=8 \Delta \mathrm{l}$. The nonlinear medium is excited by a unit amplitude pulse with a sinusoidal carrier frequency $\mathrm{f}_{\mathrm{c}}=1.3710^{14} \mathrm{~Hz}$ considering a hyperbolic secant envelope function with a characteristic time constant of 14.6 fs [7]. Fig. 1 depicts the behavior of a pulse propagating in a nonlinear medium after 12000 and 24000 iterations respectively. This shows for both numbers of iterations a stable soliton conserving its amplitude and width and another soliton with very weak amplitude. The agreement between our results and those presented in references [7, 9] and [12] is very good. Fig. 2 illustrates the variations of the normalized nonlinear admittance $\mathrm{Y}_{\mathrm{ox}}(\mathrm{t})$ of the capacitive stub of the HSCN. Fig. 3 gives the Fourier transforms of the main solitons present in the nonlinear medium after 12000 and 24000 iterations. Here again, the behavior of the numerical TLM results is similar to that presented in [7] and [9].

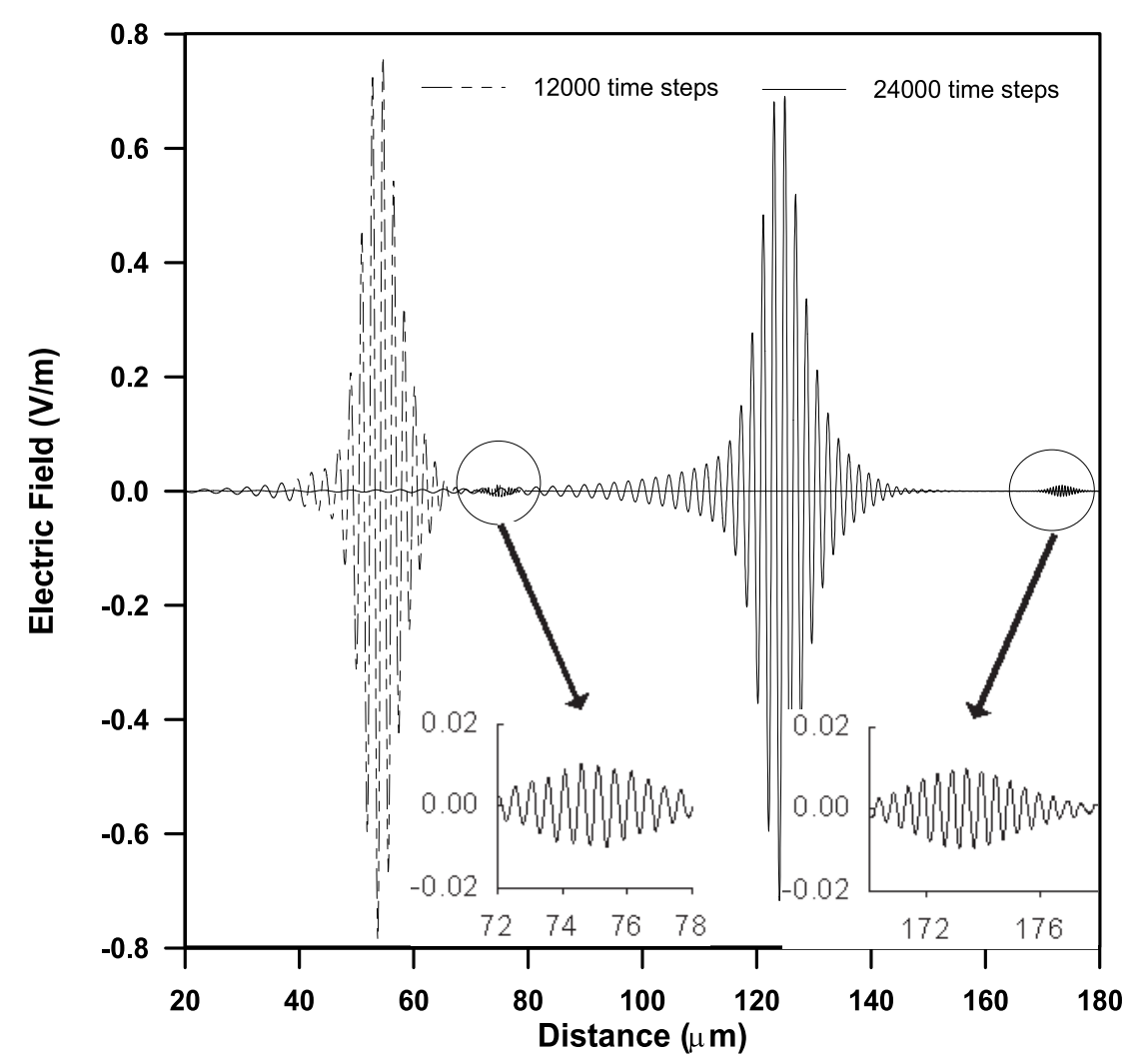

Fig. 1. TLM results of the propagation in the nonlinear optical medium after 12000 and 24000 time step. 


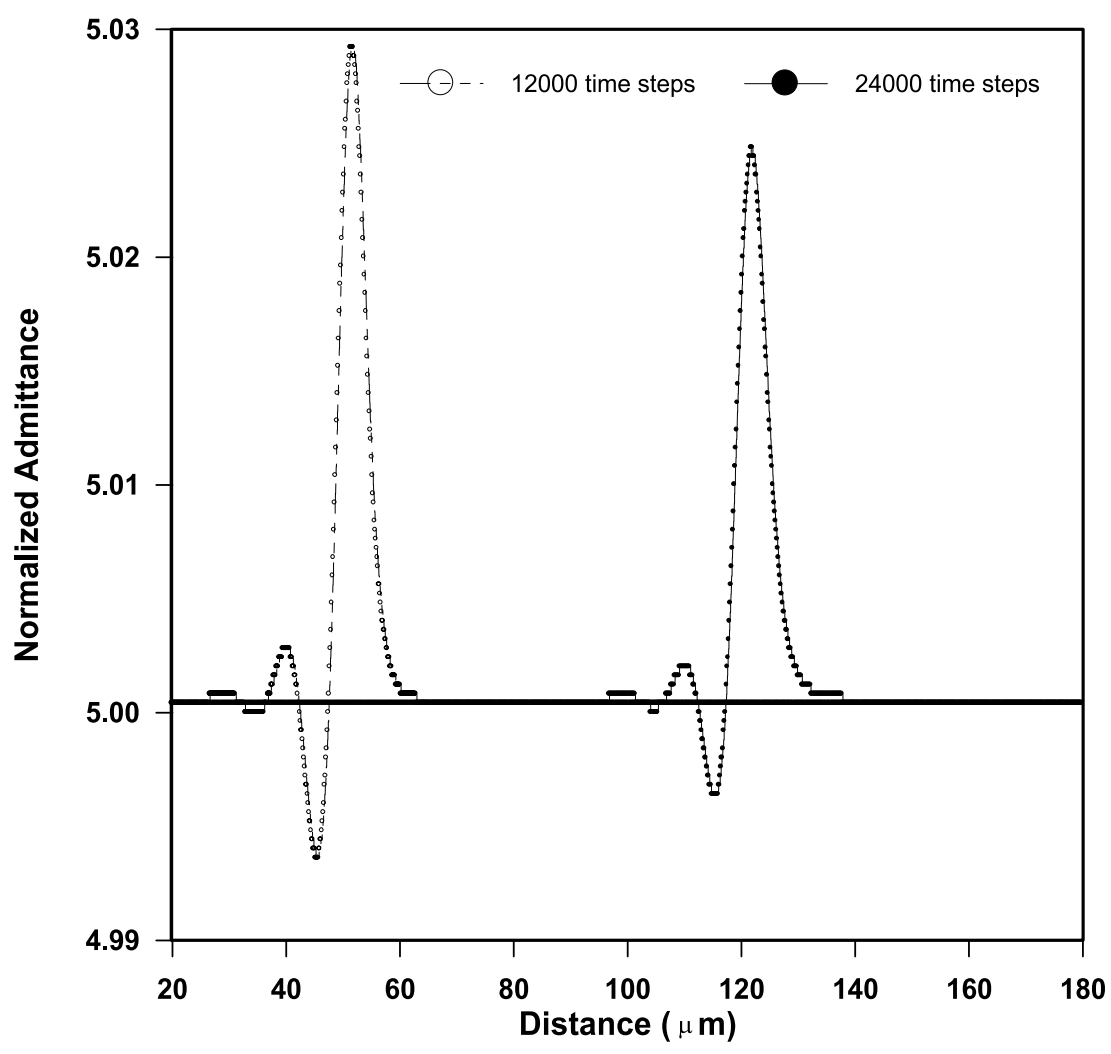

Fig. 2. The normalized admittance $Y_{\text {ox }}(t)$ of the capacitive stub of the HSCN after 12000 and 24000 time steps.

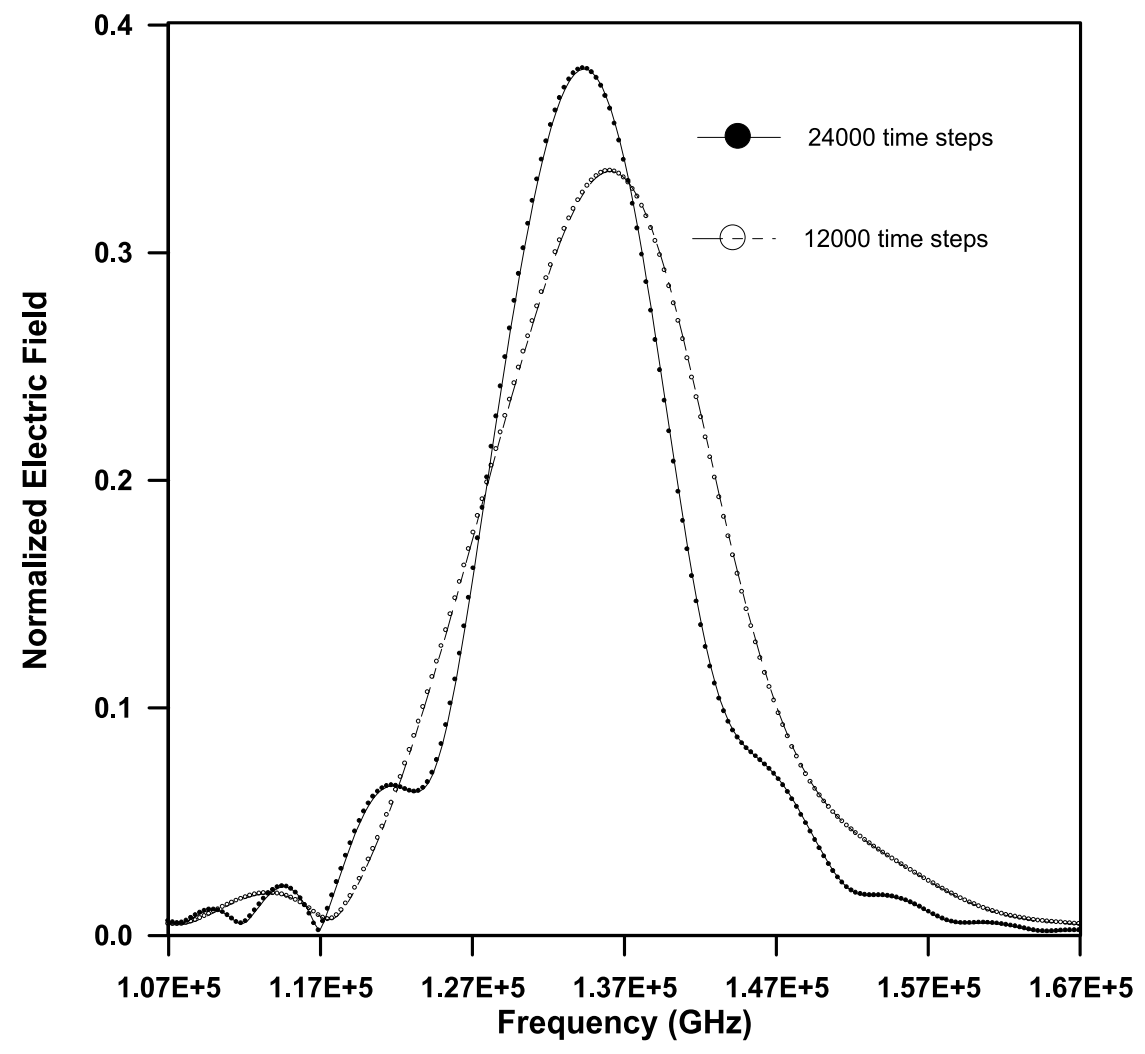

Fig. 3. Fourier spectrum of the soliton of Fig. 1. 


\section{Conclusion}

A novel HSCN-TLM approach is proposed for the simulation of the nonlinear optical media. It is based on the piecewise linear convolution discretization (PLRC) technique, the introduction of voltage sources modeling linear and nonlinear properties and the introduction of the variable admittance concept. This model has been illustrated for media with quantic effects. The obtained numerical results validate this model and prove its contribution to the development of the TLM method to model nonlinear optical media. 